Please do not remove this page

RMIT

UNIVERSITY

\title{
Blind adaptive multiuser detection for chaos CDMA communication
}

Lau, Yuu; Jusak, Jusak; Hussain, Zahir

https://researchrepository.rmit.edu.au/esploro/outputs/9921861915401341/filesAndLinks?institution=61RMIT_INST\&index=null

Lau, Y., Jusak, J., \& Hussain, Z. (2005). Blind adaptive multiuser detection for chaos CDMA communication. Proceedings of the Tencon 2005 IEEE Region 10 Conference.

https://doi.org/10.1109/TENCON.2005.300876

Published Version: https://doi.org/10.1109/TENCON.2005.300876

Repository homepage: https://researchrepository.rmit.edu.au

(c) 2005 IEEE. Personal use of this material is permitted. However, permission to reprint/republish this material for advertising or promotional purposes or for creating new collective works for resale or redistribution to servers or lists, or to reuse any copyrighted component of this work in other works must be obtained from the IEEE.

Downloaded On 2023/04/26 23:01:51 +1000 


\title{
Blind Adaptive Multiuser Detection for Chaos CDMA Communication
}

\author{
Yuu-Seng Lau, Jusak Jusak, and Zahir M. Hussain \\ School of Electrical and Computer Engineering, RMIT University, Melbourne, Victoria 3000, Australia. \\ E-mails: s9701549@student.rmit.edu.au; s3001898@student.rmit.edu.au; zmhussain@iee.org
}

\begin{abstract}
Chaos-based CDMA communication has opened up a new category for spread spectrum communication, where the spreading code that is used to scramble data in the CDMA systems can be generated using a single mathematical relationship of a chaotic generator instead of using the pseudo-noise (PN) generator. Recent studies showed that this sequence can enhance the security and increase the capacity of the system at the physical link due to its bifurcation behavior. In this paper, we investigate the performance of multiuser detection schemes in chaos based and PN code-division multiple access (CDMA) systems. Two wellknown schemes are applied and compared: adaptive filtering using the constant modulus algorithm (CMA) and matched filtering (using a correlator). Simulation results showed that, for both schemes, the chaos-based CDMA outperforms the PN-based CDMA in terms of bit error rate (BER) and mean-squared error (MSE).
\end{abstract}

\section{INTRODUCTION}

During the past decade, an intensive research in modelling non-linear dynamic systems provided a possibility to approach this non-linear system using chaos theory. Research in this area had uncovered the beauty of a mixture of deterministic and dynamic stochastic behaviors of chaotic systems. Interestingly, this has unfolded several common signal properties to the communication engineering problem, such as the use of random noise-like behavior of the chaotic system in both analog and digital spread spectrum communications [1]- [4]. In digital communications, chaotic systems are used in code-level optimization of a code-division multiple access (CDMA) and proven to be superior as compared to the classical approaches in many realistic environments [4], particularly in combatting the multiple access interference (MAI), [4] - [7].

Multiple access wireless communication systems employ different methods to multiplex different users for transmission over one wireless channel. There were two major scheme that had been widely used in the past, i.e., frequency division multiple access (FDMA) and time division multiple access (TDMA), which were used in first and second generation wireless mobile communications. FDMA systems assigned a distinct orthogonal frequency for each user, while TDMA systems employed orthogonal time slots for users. However, in the new generation wireless communications, a code division multiple access (CDMA) is preferable. Spread spectrum (SS) CDMA communication is produced by directly multiplying the user information bits (in the time domain) with a known spreading sequence code running at a much higher rate, to spread the user information over the bandwidth of the transmitted signal. In this way, it is expected that the detrimental effect of intentional jamming and interference arising from the inner-cell or outer cell users can be suppressed.

In CDMA systems, the spreading code is generated using a pseudo-random noise (PN) generator or some speciallydesigned code generator, such as, Gold code, Kasami code, Walsh Hadamard, M-sequence and OVSF code [10]. However, these generators produce repeating sequences, which lead to a very predictable fashion. Therefore, they performed unsatisfactory in terms of system capacity and security. Recent studies suggested that the use of chaos generators to generate the code sequence of CDMA systems [5]-[9] can increase the system capacity [4] and may also be used to address the security drawback in spread spectrum communication [3]. Furthermore, it is apparent that this sequence has the autoand cross- correlation properties requested by spread spectrum systems. The beauty of chaos generator bifurcation behavior can provide a security aspect to the system, where the chaotic sequence is very sensitive to the initial condition chosen. Hence, an exact initial condition value must be known at the receiver side to be able to regenerate the CDMA code for demodulation of the transmitted signal.

On the receiver side of the CDMA communication systems, conventional detectors are usually employed (e.g., RAKE/Correlator receivers, and zero-forcing detectors). However, these schemes do not provide optimal solutions, particularly for multi-user detection systems. Therefore, the progress of digital signal processing techniques in multiuser environments has considered the possibility of using adaptive filtering methods for the next generation of mobile communications [11] and [12]. This involves the development of blind adaptive systems, where training sequences are not required by the receiver. For example, in [13], the uniformly-distributed dithered signed-error constant modulus algorithm has been implemented for DS-CDMA multiuser detection. It was shown that the algorithm is able to lock each user to their specific set of minima, while retaining the averaged transient behavior of the constant modulus algorithm. For simplicity, in this paper we employ a conventional constant modulus algorithm (CMA) for blind user detection.

This paper is organized as follows: In Section II, the DS-CDMA communication systems model with $K$ users is presented. Section III describes the CDMA spreading code 


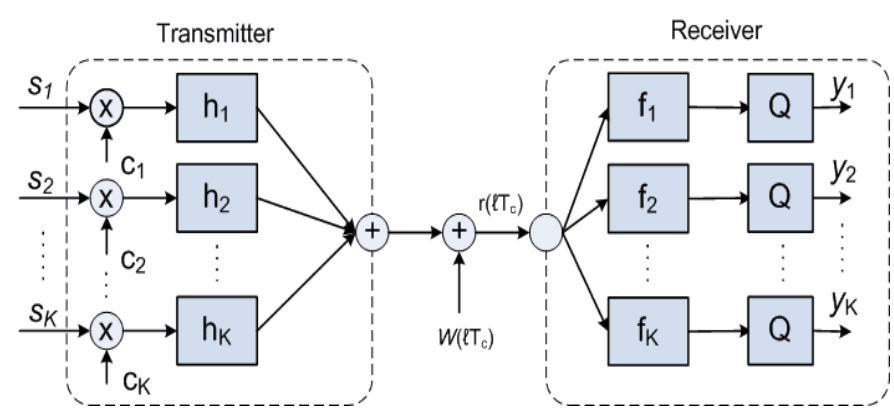

Fig. 1. DS-CDMA communication system model with $K$ users.

generation using chaos generator. Section IV briefly covers the adaptive multiuser detection techniques and a blind scheme constant modulus algorithm (CMA). Simulation results are provided in Section V. Finally, a conclusion is given in Section VI.

\section{SySTEM MODEL}

Fig. 1 depicts the transmitter and receiver system model of a baseband DS-CDMA communication system with $K$ users. Each user transmits binary symbols $s(n) \in\{-1,+1\}$ using BPSK modulation. The $k^{\text {th }}$ user of the source symbol sequence with $T_{b}$ symbol period, denoted by $s_{k}(n)$, is spread by a pseudo-noise code (or chaos code sequence) of length $L$ with a chip duration of $T_{c}$. Thus, the spreading gain of the system can be expressed as $L=T_{b} / T_{c}$. The spreading sequence of the $k^{\text {th }}$ user can be written in a vector $\mathbf{c}_{k}=\left[c_{k}(0), c_{k}(1), c_{k}(\ell), \ldots, c_{k}(L-1)\right]^{t}$, where $c_{k}(\ell) \in$ $\{-1,+1\}$ and $(.)^{t}$ is a transpose operator. The coefficients of the $k^{\text {th }}$ user channel are summarized in the vector $\mathbf{h}_{k}=$ $\left[h_{k}(0), h_{k}(1), h_{k}(\ell), \ldots, h_{k}\left(N_{h}-1\right)\right]^{t}$, where $N_{h}$ denotes the channel length. The additive Gaussian noise, $w\left(\ell T_{c}\right)$, is independent of the source symbols and has zero mean and variance $\sigma_{w}^{2}$. On the receiver part, a bank of correlators (or an adaptive filter) is used, followed by hard decision devises $(Q)$ that are applied to produce hard decision output.

\section{Chaotic Sequences}

A non-linear chaos generator used to generate the chaotic spreading sequences is given by [1], [3]:

$$
c_{n+1}=1-a c_{n}^{2} \text {. }
$$

When $a=2$, the system becomes a non-linear dynamic system. The initial value $c_{o}=c(0)$ for the system is taken from the open interval $(-1,1)$. In CDMA systems, the spreading sequences are usually in binary format. Hence, a quantization function $Q(c)$

$$
Q(c)= \begin{cases}1 & , \text { if } c>0 \\ -1 & , \text { otherwise }\end{cases}
$$

is used to convert the chaotic sequences into binary chaotic spreading code sequences. These code sequences provides a whit- noise-like auto- and cross-correlation properties as shown in Fig 2.
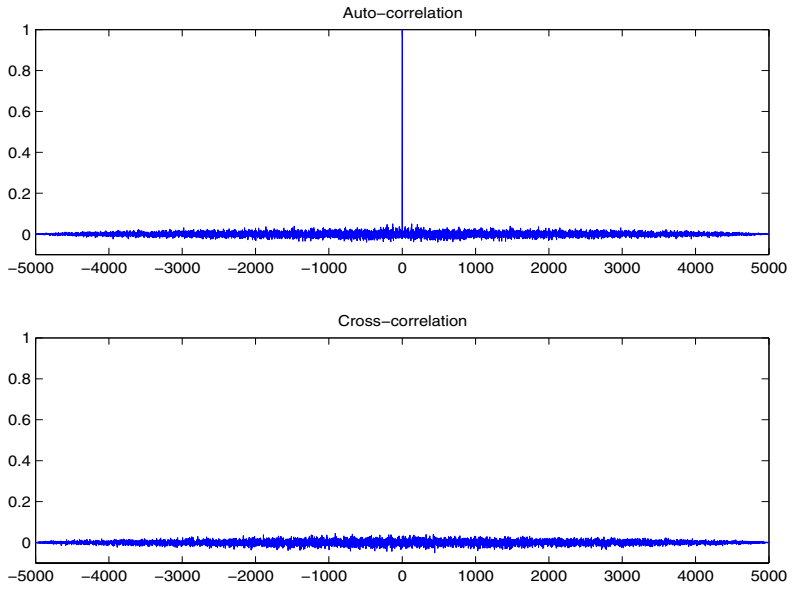

Fig. 2. Above: auto-correlation function of two chaotic sequences generated by equation 1 with two initial values $c(0)=0.07$ and $c(0)=0.07001$. Below: cross-correlation of the above two chaotic sequences.

Fig. 2 (above) shows the auto-correlation function of two chaotic sequences generated by equation 1 with two initial values $x(0)=0.07$ and $x(0)=0.07001$. Fig. 2 (below) shows the cross-correlation of the above two chaotic sequences. Despite the fact that their initial values are just slightly different, the generation of binary chaotic spreading code sequences is very sensitive to the initial condition (value). A slight difference in the initial condition will generate a totally different chaotic sequence. This is the basis for higher security.

\section{Multiuser Detection Schemes}

\section{A. Correlator Filter Detection}

The transmitted message from the $k^{\text {th }}$ user at the chip duration $\ell T_{c}$ is expressed as:

$$
\mathbf{u}_{k}\left(\ell T_{c}\right)=\mathbf{H}_{k} \mathbf{C}_{k} \mathbf{s}_{k}(n) .
$$

where $\mathbf{H}_{k}$ denotes the channel convolution matrix:

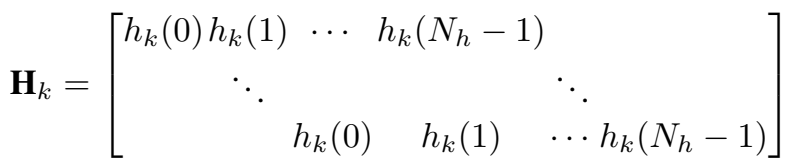

with a dimension of $N_{f} \times\left(N_{f}+N_{h}-1\right) ; N_{f}$ being the equalizer length. The spreading code matrix $\mathbf{C}_{k}$ of dimension $\left.\left(N_{f}+N_{h}-1\right) \times N_{s}\right)$ is defined as:

$$
\mathbf{C}_{k}=\left[\begin{array}{cccc}
\mathbf{c}_{k} & & & \\
& \mathbf{c}_{k} & & \\
& & \ddots & \\
& & & \mathbf{c}_{k}
\end{array}\right]
$$

where the column vector $\mathbf{c}_{k}$, is a chip sequence of length $L$. Thus, the number of source symbols from the $k^{\text {th }}$ user that are considered to estimate the output symbol at the time-index $n$ may be given as:

$$
N_{s}=\left\lceil\frac{N_{f}+N_{h}-1}{L}\right\rceil .
$$


Clearly, the source symbol vector will be as follows: $\mathbf{s}_{k}(n)=$ $\left[s_{k}(0), s_{k}(1), \ldots, s_{k}\left(n-N_{s}+1\right)\right]^{t}$.

Finally, the received signal vector $\mathbf{r}\left(\ell T_{c}\right)$ at the input of the filter can be expressed as:

$$
\begin{aligned}
\mathbf{r}\left(\ell T_{c}\right)= & \sum_{k=1}^{K} \mathbf{u}_{k}\left(\ell T_{c}\right)+\mathbf{w}\left(\ell T_{c}\right) \\
= & \sum_{k=1}^{K} \mathbf{H}_{k} \mathbf{C}_{k} \mathbf{s}_{k}(n)+\mathbf{w}_{k}\left(\ell T_{c}\right) \\
= & {\left[\begin{array}{llll}
\mathbf{H}_{1} \mathbf{C}_{1} & \mathbf{H}_{2} \mathbf{C}_{2} & \ldots & \mathbf{H}_{K} \mathbf{C}_{K}
\end{array}\right]\left[\begin{array}{c}
\mathbf{s}_{1}(n) \\
\mathbf{s}_{2}(n) \\
\vdots \\
\mathbf{s}_{K}(n)
\end{array}\right] } \\
& +\mathbf{w}\left(\ell T_{c}\right) .
\end{aligned}
$$

Assuming perfect channel estimation, the soft decision correlator detector output for each user is formulated as follows:

$$
\hat{y}_{k}(n)=\mathbf{r}^{t}\left(\ell T_{c}\right) \mathbf{H}_{k}^{*} \mathbf{C}_{k} .
$$

\section{B. Adaptive Filter Detection}

The soft linear detector output for each user is computed at symbol rate from the received vector $\mathbf{r}\left(\ell T_{c}\right)=$ $\left[r(1), r(2), \ldots, r\left(\ell T_{c}-N_{f}+1\right)\right]^{t}$ as follows:

$$
\hat{y}_{k}(n)=\mathbf{f}_{k}^{t} \mathbf{r}\left(\ell T_{c}\right) \text {. }
$$

In blind systems utilizing CMA, adaptation of the filter coefficients using the gradient descent method can be expressed as follows:

$$
\mathbf{f}(n+1)=\mathbf{f}(n)+\varphi \mathbf{r}(n) \psi_{\mathrm{cma}}\left(y_{n}\right),
$$

where $\varphi$ is a small positive step-size, and $\psi_{\mathrm{cma}}\left(y_{n}\right)$ is the CMA error function. The error function may be formulated as :

$$
\psi_{\mathrm{cma}}\left(y_{n}\right) \equiv y_{n}^{*}\left(\gamma-\left|y_{n}\right|^{2}\right),
$$

where $\gamma$ is a dispersion constant defined as $\gamma \equiv$ $E\left[\left|s_{n}\right|^{4}\right] / E\left[\left|s_{n}\right|^{2}\right]$. Under the condition of perfect blind equalization (PBE) [14], equalizers minimizing the CM cost function can perfectly recover the original source symbols for some values of the system delay $\delta$ [where $0 \leq \delta \leq N_{s}-1$ ] such that $y_{n}=s_{n-\delta}$. In the same direction, the adaptive multiuser detection filters are also considered as equalizers. Therefore, this blind adaptive algorithm may be employed to estimate the transmitted symbol sequence of each user.

It should be noted that, since the CMA cost-surface has multi-modal properties [14], a set of minimum points exists. Therefore, in a multiuser system with $K$ users, $K$ sets of minima exist. Each minimum point of a specific set of minima relates to a different delay. Depending on the channel characteristics and noise distortion, their depth can also vary widely. In this point of view, adaptive multi-user detection refers to the algorithms capability to lock one user to its set of minima and converge to those points. If the algorithm leads to the wrong set of minima, it directs the decision to the wrong user.

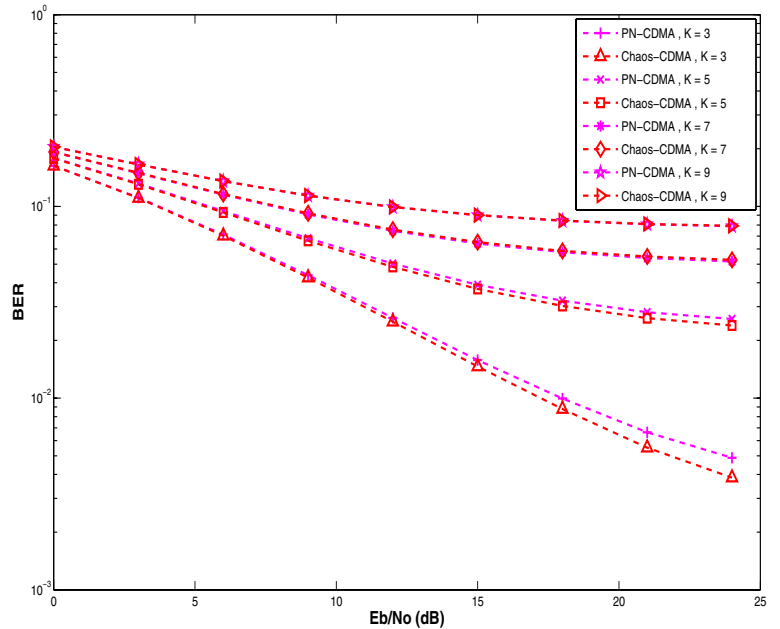

Fig. 3. BER performance of the chaos-based and PN-based CDMA with spreading gain (spreading factor) $L=16$ in a Rayleigh fading environment. The received signal is decoded using a correlator filter detector.

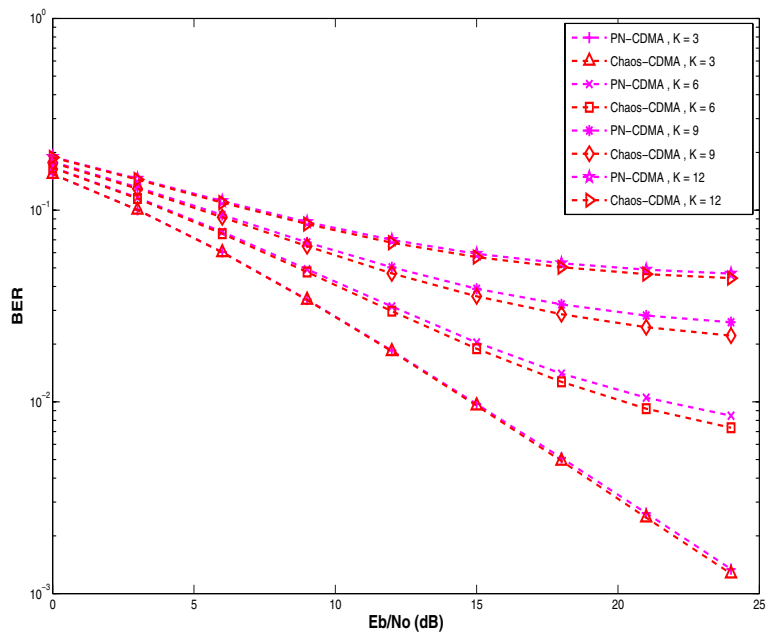

Fig. 4. BER performance of the chaos-based and PN-based CDMA with spreading gain (spreading factor) $L=32$ in a Rayleigh fading environment. The received signal is decoded using a correlator filter detector.

\section{Simulation Results}

For correlation detection, the multiuser CDMA system was simulated for different number of users in a wireless Rayleigh environment over 500 realizations. We assumed that the receiver has a perfect channel estimation. Figs. 3 - 5 show the BER performance of the chaos-based and PN-based CDMA using a correlator, with spreading gain (spreading factor) of $L=16, L=32$, and $L=64$ in a Rayleigh fading environment. Generally the chaos-based CDMA provides better BER performance than the PN-based CDMA. It is worth noting that chaos-based CDMA performance is better than that of PNbased CDMA for a lower number of multiusers at lower spreading gain (spreading factor) and for a higher number of multiusers at a higher spreading gain (spreading factor). There 


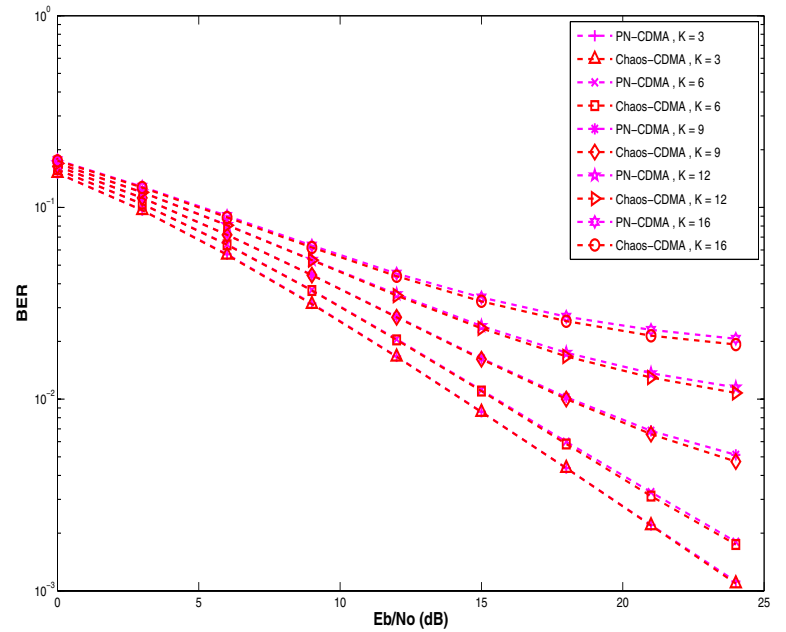

Fig. 5. BER performance of the chaos-based and PN-based CDMA with spreading gain (spreading factor) $L=64$ in a Rayleigh fading environment. The received signal is decoded using a correlator filter detector.
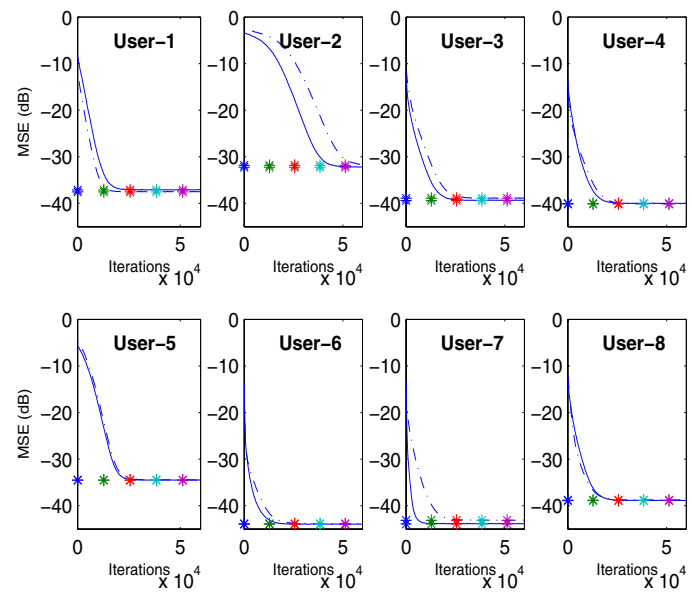

Fig. 6. Individual averaged mean-squared error (MSE) trajectories of adaptive filters. Chaos-based ( - line) and PN-based (-. line) CDMA were simulated for $K=8$ users with spreading gain (spreading factor) of $L=64$.

is no performance gain otherwise.

The adaptive multiuser detection on CDMA system was simulated for different number of users in the same cell and different spreading gains for both chaos and PN sequences. Dispersion constant $\gamma$ was set to 1 for BPSK modulation. The algorithm step-size $(\varphi)$ was set to $8 \times 10^{-4}$ when the number of users was 16 and $\varphi=5 \times 10^{-4}$ when the number of users was 8 . The filter length $N_{h}$ was set to $2 L$. Along the way from transmitter to receiver, white Gaussian noise is added and it was assumed that $\mathrm{SNR}=40 \mathrm{~dB}$. The mean-squared error trajectories in all cases were averaged over 100 iterations.

In Fig. 6, the number of users $(K)$ in the cell was set to 8 and the spreading gain of the code sequences was set to $L=64$. The figure depicts the individual mean-squared error trajectory of each user. It is shown that each user was locked at different
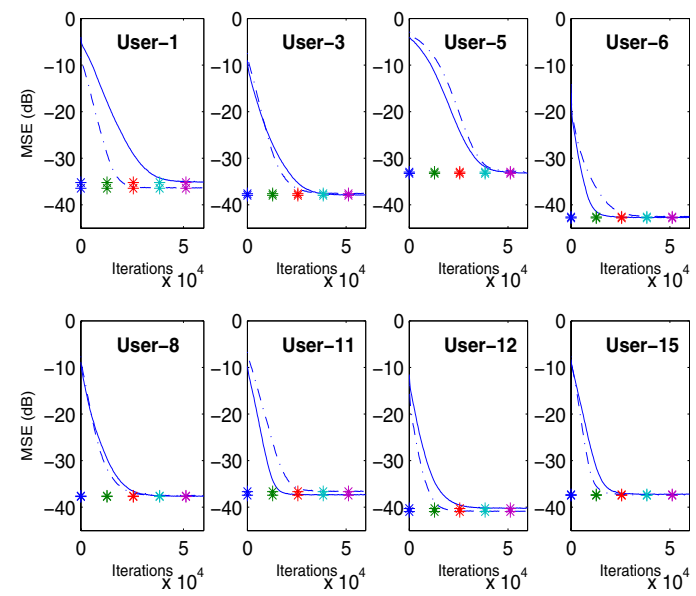

Fig. 7. Individual averaged mean-squared error (MSE) trajectories of adaptive filters. Chaos-based ( - line) and PN-based ( - . line) CDMA were simulated for $K=16$ users with spreading gain (spreading factor) of $L=64$.
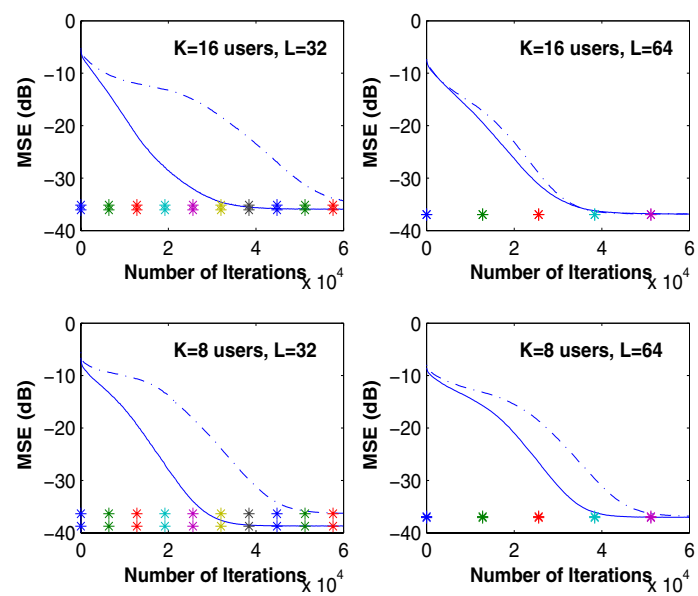

Fig. 8. Averaged mean-squared error trajectories taken from the average value of the $K$ users. Chaos-based ( - line) and PN-based (-. line) CDMA were simulated for different number $K$ of users with different values of spreading gain (spreading factor) $(L)$.

value of MSE for both sequences. The convergence speed of the filter coefficients adaptation taken from the average MSE of all users for different spreading codes is plotted in Fig. 8 (bottom). It can be seen clearly that CDMA system utilizing chaos sequences outperforms the PN-based CDMA in terms of convergence speed, while providing the same MSE for chaos and PN code sequences.

Increasing the number of users in the cell to $K=16$ gives the same performance as above. The averaged MSE trajectories for individual users are shown in Fig. 7, while the average MSE of all users for different spreading codes are plotted in Fig. 8 (top). 


\section{CONCLUSION}

The use of blind adaptive constant modulus algorithm for multiuser detection is studied, where the transmitted signal is spread by chaos-based sequences for CDMA. A comparison with PN-based CDMA in multiuser detection showed that the chaos-based CDMA sequences do provide enhancement in terms of BER and MSE for both detection schemes (matched filtering/correlator detection and adaptive filter detection).

\section{REFERENCES}

[1] F. C. M. Lau, C. K. Tse, M. Ye, and S. F. Hau, "Coexistence of chaosbased and conventional digital communication systems of equal bit rate," IEEE Trans. Circuits and Systems, vol. 51, pp. 391-408, Feb. 2004.

[2] M. P. Kennedy and G. Kolumban, "Digital communications using choas," Elsevier Signal Processing Journal, vol. 80, pp. 1307-1320, 2000.

[3] Y.-S. Lau and Z. M. Hussain, "A new approach in chaos shift keying for secure communication," IEEE International Conference on Information Technology and Applications 2005, Sydney, Australia, July 2005.

[4] R. Rovatti, G. Mazzini, and G. Setti, "On the ultimate limits of chaosbased asynchronous DS-CDMA I : Basic Definitions and Results" IEEE Trans. Circuit and Systems, vol. 51, pp. 1336-1347, July 2004.

[5] R. Rovatti and G. Mazzini "Interference in DS-CDMA systems with exponentially vanishing autocorrelations: Chaos-based spreading is optimal," Electron. Lett., vol. 34, 1998.
[6] G. Mazzini, R. Rovatti, and G. Setti, "Interference minimization by autocorrelation shaping in asynchronous DS-CDMA systems: Chaos-based spreading is nearly optimal," Electron. Lett., vol. 35, 1999.

[7] T. kohda and H. Fujisaki, "Variances of multiple access interference code average agianst data average," Electron. Lett, vol. 36, 2000.

[8] H.-B. Ghobad and C. D. McGillem, "A chaotic direct-sequence spreadspecturm communication system," IEEE Trans. Communications, vol. 42, no. 234, pp. 1524- 1527, Feb/Mar/Apr. 1994.

[9] T. Kohda and A. Tsuneda, "Even- and odd-correlation fucntions of chaotic chebyshev bit sequences for CDMA," in Proc. IEEE Int. Symp. Spread Specturm Technology and Applications, pp. 391-395, 1994.

[10] M. Ibnkahla, Signal Processing for Mobile Communications Handbook, CRC Press, ch. 10, 2005.

[11] S. Moshavi, "Blind Adaptive Multiuser Detection," IEEE Tran. on Information Theory, vol. 41, no. 4, pp. 944-960, July 1995

[12] M. Honig and M. K. Tsatsanis, "Adaptive Techniques for Multi-user CDMA Receivers," IEEE Signal Processing Magazine, vol. 34, pp. 4961, May 2000.

[13] J. Jusak and Z. M. Hussain, "Low Complexity Blind Adaptive MultiUser Detection for Up-link DS-CDMA," Australian Telecommunications, Networks and Applications Conference (ATNAC), Sydney, Australia, 2004.

[14] C. R. Johnson, Jr., P. Schnitter, T. J. Endres, et al., "Blind Equalization Using the Constant Modulus Criterion: A Review," IEEE Proceedings, vol. 86, No. 10, pp. 1927-1950, October 1998. 